\title{
Deficient Mismatch Repair and Lymphocytic Response to Tumor as Prognostic Markers in Stage II Colon Cancer Patients
}

\author{
Murat Sari ${ }^{1}$, Elif Atag $^{1}$, Tarik Demir ${ }^{1}$, Eda Tanrikulu Simsek ${ }^{1}$, Ezgi Coban ${ }^{1}$ and Makbule Cikrikcioglu ${ }^{2}$ \\ ${ }^{1}$ Department of Medical Oncology, Haydarpasa Numune Research and Training Hospital, Istanbul, Turkey \\ ${ }^{2}$ Department of Pathology, Haydarpasa Numune Research and Training Hospital, Istanbul, Turkey
}

\begin{abstract}
Objective: To investigate the relationship between colon cancer (CC) subtypes defined by the status of tumor-infiltrating lymphocytes (TIL) and mismatch repair (MMR) combination with clinicopathological features and survival.

Study Design: Observational study.

Place and Duration of Study: Department of Medical Oncology, Haydarpaşa Numune Research and Training Hospital, Istanbul, Turkey, from July 2010 to March 2020.

Methodology: Eighty-three patients with operated stage II colon cancer were included in the study. Pathology, surgery and oncological treatment and follow-up information were obtained from patient files; and statistical analyses were performed on overall survival (OS). Tumor-infiltrating lymphocytes and mismatch repair status was determined with the help of immunohistochemistry. Results: TIL-high and deficient MMR (dMMR) status were detected in 26 patients (31.3\%) and 21 patients (25.3\%), respectively. Tumors were divided into four subgroups according to TIL and MMR status. TIL-high/dMMR tumors had the most favourable prognosis, while TIL-low/proficient MMR tumors exhibited poor OS.

Conclusion: The combination of TIL and MMR could enable us to differentiate patients' survival outcomes in more details. Therefore, considering that the TIL and MMR status, evaluated by IHC, may be a cost-effective and effective option for risk classification in patients with stage II colon cancer.
\end{abstract}

Key Word: Lymphocytic response, Mismatch repair, Prognosis, Tumor-infiltrating lymphocytes, Stage II cancer, Colon cancer.

How to cite this article: Sari M, Atag E, Demir T, Simsek ET, Coban E, Cikrikcioglu M. Deficient Mismatch Repair and Lymphocytic Response to Tumor as Prognostic Markers in Stage II Colon Cancer Patients. J Coll Physicians Surg Pak 2022; 32(02):186-192.

\section{INTRODUCTION}

Colon cancer (CC) was the fourth most commonly diagnosed cancer with 1.1 million new cases in 2020, and the fifth most common cause of cancer-related deaths with 551,000 deaths. ${ }^{1}$ Stage II disease accounts for about $25 \%$ of all colon cancer cases. In stage II colon cancer, the 5-year disease-specific survival (DSS) rate was $84.7 \%$, and the 5 -year overall survival (OS) rate was $70.3 \%{ }^{2}$

While adjuvant chemotherapy is standard for stage III CC, its utility for stage II disease is controversial; and appears to provide a maximum of $5 \%$ benefit in 5 -year survival. Adjuvant chemotherapy may be considered for stage II CC with high-risk features. ${ }^{3}$

Correspondence to: Dr. Murat Sari, Department of Medical Oncology, Haydarpasa Numune Research and Training Hospital, Istanbul, Turkey

E-mail: drmuratsari@gmail.com

Received: July 07, 2021; Revised: November 09, 2021;

Accepted: December 20, 2021

DOI: https://doi.org/10.29271/jcpsp.2022.02.186
There is no clear evidence that adjuvant treatment, according to these markers, is beneficial in terms of relapse or death. ${ }^{4}$ Therefore, markers are needed to predict the treatment response and prognosis that can be easily applied to routine care in the clinical decision-making process.

Tumor lymphocytic reaction, evaluated by histopathological examination for colon cancer, has been shown to be an important prognostic biomarker even after the effects of prognostic factors, such as stage and lymph node number are cleared..$^{5-7}$ Immunoscore (evaluating TIL status) has been demonstrated to give the relatively highest contribution among all other clinical variables including TNM stage, to determine the prognosis of patients with stage I-III colon cancer. ${ }^{8}$ Unfortunately, the determination of the immunoscore usually requires extra effort and time because of needing for further immunohistochemistry (IHC) staining. This complicates the general use of this scoring system.

TIL status is determined using routine sections stained with hematoxylin and eosin (HE) as measured by Klintrup-Mäkinen ( $\mathrm{K}$ M) grading, ${ }^{9}$ is a significant prognostic marker for colorectal cancer survival. ${ }^{5,10}$ However, the effect of TIL status determined by the K-M grading system on stage II CC prognosis has not been studied properly. 
Approximately 15 to 20 percent of colorectal cancers have a deficiency of mismatch repair (dMMR) determined by microsatellite instability (MSI) analysis or immunohistochemistry for DNA mismatch repair proteins (most commonly MLH1 or MSH2). dMMR is more prevalent in stage II CC than stage III (21 vs. 14 percent in one study). ${ }^{11}$ dMMR is a prognostic biomarker for stage II CC patients since it shows a low risk of recurrence.

As a result, local immune-related tumor responses, such as TIL and conditions showing the host's DNA repair status, such as MMR status, have prognostic value, respectively. To the authors' knowledge, very few studies have evaluated these two mechanisms at the same time in evaluating tumor prognosis. ${ }^{12}$ It is also unclear whether the combination of these two separate mechanisms will provide extra useful prognostic information in stage II CC patient.

The aim of this study was to investigate the relationship between CC subtypes defined by the status of TIL and MMR combination with clinicopathological features and survival in stage IICC patients.

\section{METHODOLOGY}

Eighty-three pathological T3NO (stage II) CC patients who were followed up in Haydarpaşa Numune Research and Training Hospital, Istanbul, Turkey, between July 2010 and March 2020, were included in this study. Inclusion criteria were: histopathologically confirmed stage II colon cancer, according to the $7^{\text {th }}$ edition of the American Joint Committee on Cancer TNM classification, curative RO resection and lymphadenectomy, and patients with enough pathologic slides to detect MMR and TIL status. Exclusion criteria were: tumors of the rectosigmoid junction or rectum, patients $\leq 18$ years of age, patients who underwent an emergency surgery or palliative surgery for obstruction or perforation, and individuals with hereditary colon carcinoma syndromes.

All patients were followed up by standard protocols with carcinoembryonic antigen (CEA) level testing, and chest, abdomen, and pelvis computed tomography scan. Demographic information, pathological features of the tumor, stage at diagnosis, duration and type of adjuvant therapy were obtained from patient records. The last date of follow-up was considered either the date of death or the date of the last visit. Survival status of patients obtained from patient files, and from the Ministry of Health, Death Registry System, Republic of Turkey.

This study was conducted under the ethical consideration of the 1964 Helsinki Declaration and the Institutional Research Committee.

HE-stained pathological slides of 83 CC patients were evaluated in terms of histological type, lymphovascular and perineural invasion, grade, mucinous histology, number of lymph nodes examined and, tumor border by a gastrointestinal pathologist, who is unaware of patients' clinical information.
As a result of evaluating HE painted slides, TIL status was decided and scored according to K-M grading. ${ }^{5,9,13}$ TIL density was scored in tumor-rich areas consisting of $>60 \%$ neoplastic cells. Only lymphocytes that entered the tumor epithelium were counted for evaluation, while stromal lymphocytes were not evaluated. The mean TIL value per hpf was obtained by dividing the total number of TILs by 5 for each tumor. Tumors were grouped as TIL-high ( $\geq 2$ TIL/hpf) and TIL-low ( $<2$ TIL/hpf) according to the cut-off value. The deficiency or proficiency of the MMR status (dMMR and pMMR) was determined by evaluating the expression of the four key proteins (MSH2, MSH6, MLH1, and PMS2) by immunohistochemical (IHC) analysis. The expression loss of one or more of these proteins was considered as dMMR. ${ }^{14}$

SPSS, version 25.0 (IBM SPSS, Chicago, IL, USA) was used for statistical analysis. Tumors were divided into four subgroups as TIL-high/dMMR, TIL-high/pMMR, TIL-low/dMMR, and TILlow/pMMR, according to TIL and MMR status. By keeping the MMR variable constant, demographic and tumor characteristics of the patients were compared according to the changing TIL status. Differences between groups were analysed using the Chi-square test (or Fisher's Exact test as appropriate) for categorical (dichotomous) parameters, which were described using frequencies and percentages. Continuous variables were presented as the mean \pm standard deviation and were analysed using the Student's t-test. Survival curves were obtained with the help of the Kaplan-Meier method and compared with the logrank test. OS was determined from the date of surgery until the date of last follow-up or death. The last contact date was used as the censorship date for patients still alive at the end of follow-up. Cox regression analysis was used to perform univariate and multivariate analysis. Variables that significantly affect OS or those with a $p$-value $<0.2$ were evaluated in the multivariate analysis. A p-value $<0.05$ was considered statistically significant.

\section{RESULTS}

Eighty-three stage II CC patients were included in the study. The mean age of the patients was $66 \pm 12.5$ years. Mean followup time was 60 months (78.6 for dMMR and 52.3 for pMMR; $p$ $<0.001$ and 68 for TIL-high and 54.8 for TIL-low; $p<0.001$ ). Approximately $22 \%$ patients had mucinous histology and $11 \%$ had high-grade tumors. The clinicopathological features of 83 patients in the study are summarised in Table I.

TIL-high and dMMR status were detected in 26 patients (31.3\%) and 21 patients (25.3\%), respectively. Relationships between the variables and the combination of MMR/TIL status are summarised in Table I. The Splenic flexure was accepted as the anatomical border in separating the right and left colon tumors (39 patients; $47 \%$ right tumors) and included in the left colon. A significant relationship between dMMR status and high-grade and mucinous histology was detected, and also the relationship between dMMR and TIL-high status tended to be signifi$\operatorname{cant}(p=0.06)$. 
Table I: Clinical and pathological characteristics of 83 patients with stage II colon cancer according to TIL/MMR status.

\begin{tabular}{|c|c|c|c|c|c|c|}
\hline & $\begin{array}{l}\text { TIL-low/pMMR } \\
\mathrm{n}=\mathbf{4 6}\end{array}$ & $\begin{array}{l}\text { TIL-high/pMMR } \\
\mathrm{n}=16\end{array}$ & p-value & $\begin{array}{l}\text { TIL-low/dMMR } \\
\mathrm{n}=11\end{array}$ & $\begin{array}{l}\text { TIL-high/dMMR } \\
\mathrm{n}=10\end{array}$ & p-value \\
\hline \multicolumn{7}{|l|}{ Age (years) } \\
\hline Mean \pm SD & $65.8 \pm 12.4$ & $67 \pm 14.5$ & 0.757 & $67.5 \pm 11.3$ & $64.1 \pm 12.5$ & 0.516 \\
\hline \multicolumn{7}{|l|}{ Gender } \\
\hline Male & $26(56.5)$ & $7(43.8)$ & 0.378 & $6(45.5)$ & $6(60)$ & 1.000 \\
\hline Female & $20(43.5)$ & $9(56.2)$ & & $5(54.5)$ & $4(40)$ & \\
\hline \multicolumn{7}{|l|}{ Site } \\
\hline Right & $19(41.3)$ & $8(50)$ & 0.546 & $7(63.6)$ & $5(50)$ & 0.670 \\
\hline Left & $27(58.7)$ & $8(50)$ & & $4(36.4)$ & $5(50)$ & \\
\hline \multicolumn{7}{|l|}{ Grade } \\
\hline Low grade & $42(91.3)$ & $15(100)$ & 0.564 & $9(81.8)$ & $7(70)$ & 0.635 \\
\hline High grade & $4(8.7)$ & $0(0)$ & & $2(18.2)$ & $3(30)$ & \\
\hline \multicolumn{7}{|l|}{ Mucinous } \\
\hline No & $39(84.8)$ & $12(80)$ & 0.696 & $8(72.7)$ & $5(50)$ & 0.387 \\
\hline Yes & $7(15.2)$ & $3(20)$ & & $3(27.3)$ & $5(50)$ & \\
\hline \multicolumn{7}{|c|}{ Lymphovascular invasion } \\
\hline No & $42(91.3)$ & $16(100)$ & 0.565 & $8(72.7)$ & $8(80)$ & 0.550 \\
\hline Yes & $4(8.7)$ & $0(0)$ & & $3(27.3)$ & $2(20)$ & \\
\hline \multicolumn{7}{|c|}{ Perineural invasion } \\
\hline No & $42(91.3)$ & $15(93.8)$ & 1.000 & $11(100)$ & $10(100)$ & \\
\hline Yes & $4(8.7)$ & $1(6.2)$ & & $0(0)$ & $0(0)$ & \\
\hline \multicolumn{7}{|l|}{ Adjuvant } \\
\hline No & $36(80)$ & $16(100)$ & 0.096 & $9(81.8)$ & $9(90)$ & 1.000 \\
\hline Yes & $9(20)$ & $0(0)$ & & $2(18.2)$ & $1(10)$ & \\
\hline \multicolumn{7}{|l|}{ Relapse } \\
\hline Yes & $8(17.4)$ & $1(6.3)$ & 0.425 & 0 & 0 & \\
\hline No & $38(82.6)$ & $15(93.7)$ & & 11 & 10 & \\
\hline \multicolumn{7}{|l|}{ Living status } \\
\hline Alive & 35 & 15 & 0.160 & 10 & 9 & 1.000 \\
\hline Exitus & 11 & 1 & & 1 & 1 & \\
\hline
\end{tabular}

Table II: Overall survival data in terms of MMR, TIL status and tumor high-risk features.

\begin{tabular}{|l|l|l|}
\hline & & p-value \\
\hline Mean OS of the cohort (months) & $96.1(86.1-106)$ & \\
Perineural invasion present & $98.7(88.9-108.6)$ & $<0.001$ \\
Perineural invasion absent & $36.8(9.3-55.1)$ & \\
\hline dMMR mean OS & $110(101-118)$ & \\
5-year overall survival rate & $100 \%$ & 0.013 \\
pMMR mean OS & $69(63-75)$ & \\
5-year overall survival rate & $83 \%$ & \\
\hline TIL-high mean OS & $109.3(99.6-119.1)$ & \\
5-year overall survival rate & $96 \%$ & 0.026 \\
TIL-low mean OS & $71.4(65.5-77.5)$ & \\
5-year overall survival rate & $85 \%$ & 0.031 \\
\hline pMMR/TIL-low mean OS & $67(59.7-74.3)$ & \\
pMMR/TIL-high mean OS & $81(77.4-84.5)$ & \\
dMMR/TIL-low mean OS & $69.1(63.8-74.5)$ & \\
dMMR/TIL-high mean OS & $112.6(104.9-120.4)$ & \\
\hline dMMR, deficient mismatch repair; pMMR, proficient-MMR; TIL, tumour- \\
infiltrating lymphocyte.
\end{tabular}

However, no significant relationship was found between dMMR status and female gender, proximal tumor location, and older age. Tumors were divided into four subgroups according to status of TIL and MMR; TIL-high/dMMR (12\%, 10 of 83 ), TILhigh/pMMR (19.3\%, 16 of 83), TIL-low/dMMR (13.3\%, 11 of 83$)$, and TIL-low/pMMR (55.4\%, 46 of 83 , Table I).
Fourteen patients (16.8\%) died. Of these, two patients with dMMR (one TIL-high and the other TIL-low) died due to non-cancer-related causes; the remaining 12 patients were pMMR ( 1 of them was TIL-high and non-cancer-related, and the other 11 TIL-low and 3 of them died due to non-cancer and eight died due to cancer progression).

The mean OS was 96.1 months (86.1-106 months; median overall survival could not be calculated because less than $50 \%$ of all patients died). Patients with dMMR had significantly better OS than patients with pMMR; mean OS was 110 months (95\% $\mathrm{Cl}, 101-118$ months) for the dMMR group, while it was 69 months for the pMMR group $(95 \% \mathrm{Cl}, 63-75$ months; HR, 0.09; $95 \% \mathrm{Cl}, 0.01-0.82 ; p=0.01$, Figure 1, Table II). TIL-high patients were found to have significantly better OS compared to TIL-low patients; mean OS was 109.3 months (95\% Cl, 99.6-119.1 months) for the TIL-high group compared with those who had TIL-low with a mean OS of 71.4 months $(95 \% \mathrm{Cl}, 65.5-77.5$ months; HR, 0.13; $95 \% \mathrm{Cl}, 0.01-1.06 ; \mathrm{p}=0.02$, Figure 1 , Table II).

TIL status classified both dMMR and pMMR tumors in terms of OS; TIL-high tumors had better survival data than TIL-low tumors ( $p=0.03$, Table II and Figure 2 ). 


\begin{tabular}{|c|c|c|c|c|c|c|}
\hline \multirow{2}{*}{ Variable } & \multicolumn{3}{|c|}{ Unvariable } & \multicolumn{3}{|c|}{ Multivariable } \\
\hline & HR & $95 \% \mathrm{Cl}$ & p-value & HR & $95 \% \mathrm{Cl}$ & p-value \\
\hline Gender & & & 0.368 & & & \\
\hline Male & 1.000 & $0.21-1.77$ & & & & \\
\hline Female & 0.614 & & & & & \\
\hline MMR status & & & $0.033^{* *}$ & & & $0.046 * *$ \\
\hline pMMR & 1.000 & $0.01-0.82$ & & 1.000 & $1.03-81.84$ & \\
\hline dMMR & 0.096 & & & 9.214 & & \\
\hline TIL status & & & 0.057 & & & \\
\hline Low & 1.000 & $0.01-1.06$ & & & & \\
\hline High & 0.136 & & & & & \\
\hline Perineural invasion & & & $0.001^{*}$ & & & $0.004 * *$ \\
\hline No & 1.000 & $2.58-43.05$ & & 1.000 & $1.90-31.77$ & \\
\hline Yes & 10.546 & & & 7.788 & & \\
\hline Lymphovascular invasion & & & 0.771 & & & \\
\hline Right & 1.000 & $0.27-5.60$ & & & & \\
\hline No & 1.249 & & & & & \\
\hline Tumour location & & & 0.540 & & & \\
\hline Right & 1.000 & $0.48-4.04$ & & & & \\
\hline No & 1.395 & & & & & \\
\hline Mucinous component & & & 0.884 & & & \\
\hline No & 1.000 & $0.32-3.66$ & & & & \\
\hline Yes & 1.094 & & & & & \\
\hline Adjuvan chemotherapy & & & 0.355 & & & \\
\hline No & 1.000 & $0.00-37.24$ & & & & \\
\hline Yes & 0.039 & & & & & \\
\hline High grade & & & 0.924 & & & \\
\hline No & 1.000 & $0.20-4.25$ & & & & \\
\hline Yes & 0.928 & & & & & \\
\hline
\end{tabular}

TIL-low/pMMR tumors had the most unfavourable prognosis, while TIL-high/dMMR tumors exhibited the best OS. Interestingly, OS data for TIL-high/pMMR and TIL-low/dMMR tumor subtypes were the same.

Our patients in the study were evaluated in terms of OS, according to the previously determined tumor high-risk features in terms of prognosis for Stage II CC, and only the presence of perineural invasion was found to affect OS negatively $(p<0.001$, Table III).

Variables that significantly affect OS or those with a $p$-value $<0.2$ were TIL-high status, dMMR and perineural invasion and they were evaluated in the multivariable model. It was observed that dMMR lost its significance as the perineural invasion was removed from the model while the non-significant variables were gradually removed from the model (Table III).

\section{DISCUSSION}

In this study, the CC tumors were divided into four groups according to their TIL and MMR status. Thus, tumor subtypeswere identified, which have direct relationships with OS and are independent of the tumor stage and the clinicopathological features of patients. Patients with TIL-high/dMMR tumors had the best prognosis for OS, while patients with TIL-low/pMMR tumors had the worst prognosis. Thus, this result in the study was consistent with previous studies ${ }^{12,15}$ reported on stage I-IV CRCs previously. It was confirmed that the TIL status showed the prognostic value in both $\mathrm{dMMR}$ and pMMR cases.

Interestingly, OS data for TIL-high/pMMR and TIL-low/dMMR tumor subtypes were the same. It is known that dMMR tumors usually have intense lymphocytic infiltration due to high mutational loads, ${ }^{16}$ but some may have poor results as they can somehow avoid the immune system. Conversely, although the lymphocytic response is not evident in pMMR tumors, better results can be seen in some subtypes due to increased immunogenicity. Perhaps these two situations may have caused this result, seen in these patients.

In this cohort, it was found that tumors with dMMR (25.3\%) and it was similar compared to previous meta-analysis ${ }^{17-19}$ and suggests that it reflects the normal clinical situation. 

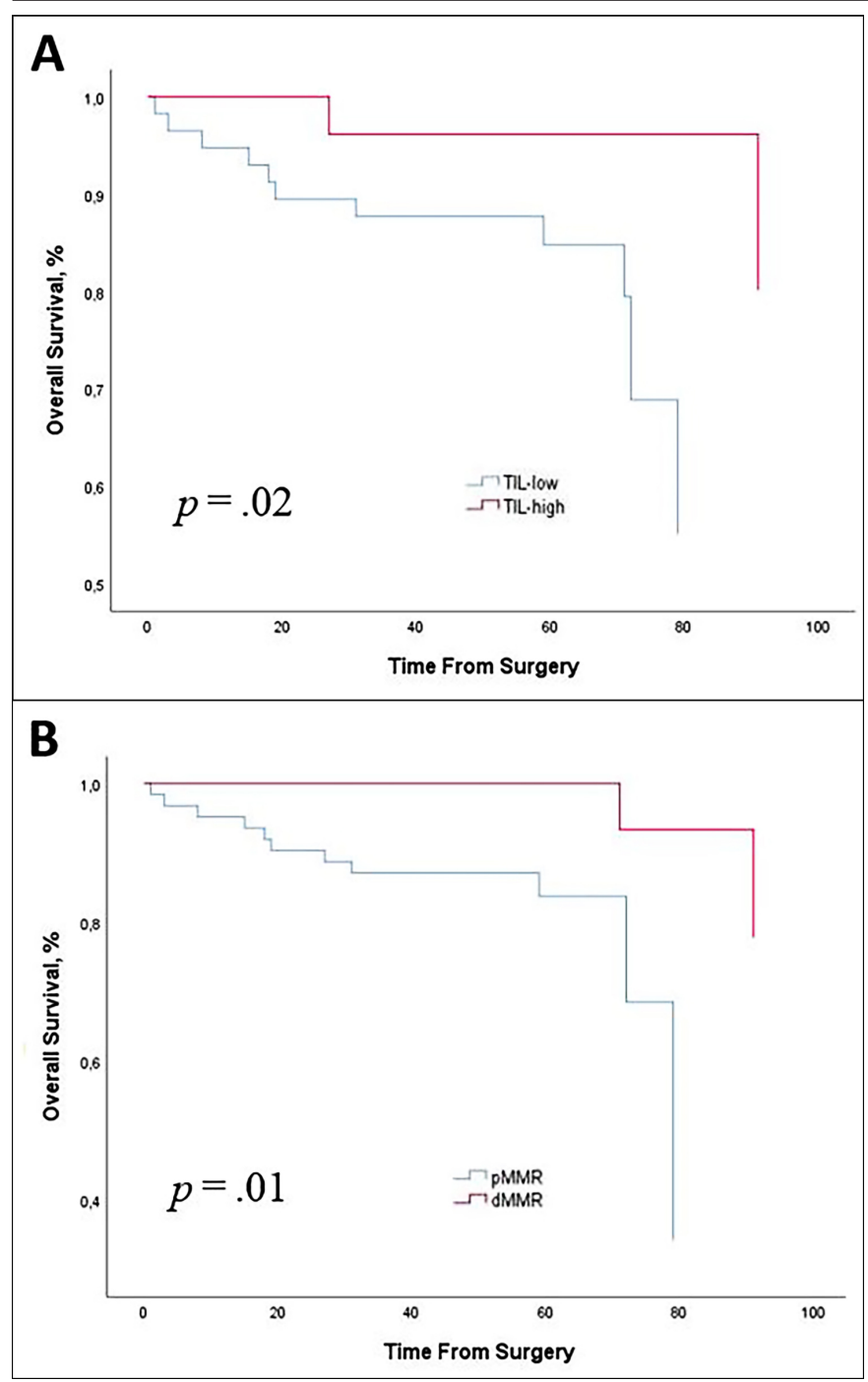

Figure 1: Kaplan-Meier plots for overall survival in patients with stage II colon cancer according to (A) TIL status and (B) MMR status.

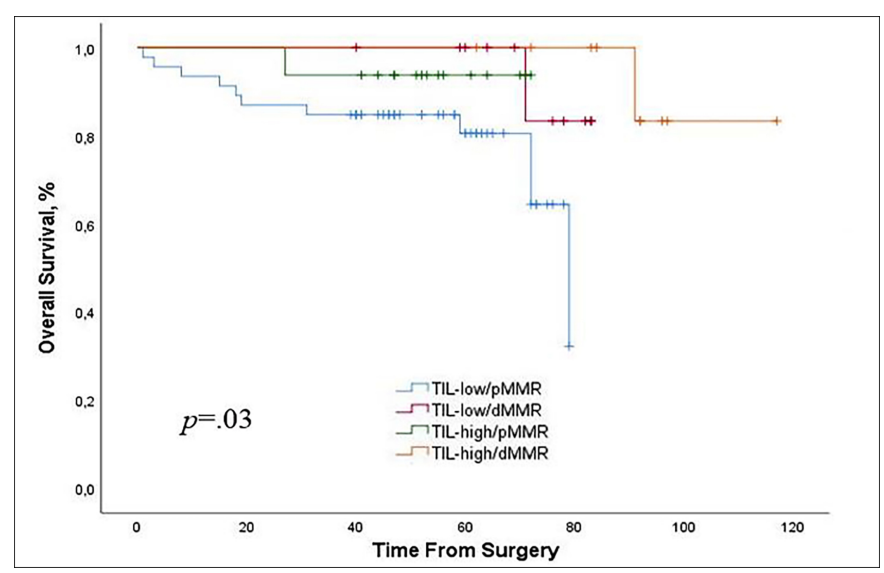

Figure 2: Kaplan-Meier plots for overall survival in patients with stage II colon cancer according to TIL/MMR status.

The rate of mucinous histology in the present study (22\%) was slightly higher compared to other studies. ${ }^{12,17,20}$ The reason for this situation is that the patients are relatively older and the right-sided CC (47\%) ratio is higher.
In this study, there was a significant relationship between dMMR and TIL-high status and overall survival in stage II CC patients and this was compatible with a published meta-analysis. ${ }^{18}$ Although stage II patients have relatively good prognosis, it was found that both dMMR and TIL-high status are effective for OS, even in patients with such a good prognosis.

In this study, approximately $14 \%$ of patients were given adjuvant therapy, and this rate was lower in the literature compared to studies with this group of patients. The reason for this situation was that the MMR and TIL status of the patients were known before treatment and the treatment of the patients was individualised, according to these results without causing any negative effects on survival. Thus, by improving patient selection, we were able to avoid giving unnecessary treatment to patients and exposing patients to the harmful side effects of adjuvant therapy.

Another important secondary finding was that we could independently demonstrate and confirm the negative effect of perineural invasion, one of the classic high-risk features, on poor survival. ${ }^{21}$

Although treatment guidelines, like National Comprehensive Cancer Network, do not recommend the use of TIL and MMR in determining the prognosis in stage II CC, as a result of the results in our study and supporting studies in the literature, the combined use of TIL and MMR status is a robust prognostic marker, independent of the stage of the disease and the high clinical risk characteristics. Moreover, the ability to examine TIL and MMR status with simple methods in routine pathology samples offers the opportunity to be widely used in determining the prognosis of patients.

Important strengths of this study are including a homogenous group of patients (PT3NO stage II CC), evaluation and scoring of tumor TIL status by an expert gastrointestinal pathologist, and long-term follow-up data of cases. To this knowledge, the relationship of TIL/MMR status classification to prognosis has not been evaluated in a cohort including only pT3NO stage II CC.

In addition to the strengths of this study, it also has some limitations. First, the number of patients is low and the cohort could not be derived from a randomised controlled clinical trial. The second is the absence of the necessary immunohistochemistry data in determining the subtypes of $T$ cell populations. Thirdly, time to progression data is missing. Interestingly, there was no relapsing patient in the dMMR group and only nine patients relapsed and all of them were from PMMR group (eight of them were TIL-low and one of them was TIL-high); but any significant relationship could not be found $(p>0.05)$. Since very few patients have relapsed, advanced statistical analysis could not be performed and, finally, the probability that confounding factors such as other molecular events or epidemiological factors may have affected survival differences seen in the TIL/MMR subtypes, and their effects could not be eliminated. 
With the publication of the results of KEYNOTE-177 this year, pembrolizumab has now taken its place in the first-line treatment of metastatic MSI-H-dMMR colon cancer. ${ }^{22}$ However, the results of this study need careful analysis. The patients in the pembrolizumab arm progressed as the best response after first-line treatment (29.4\% vs.12.3\%). In the first six months of the study, which are used to seeing from other immunotherapy studies, poor performance is obtained with these agents. This shows that even in the case of MSI-H-dMMR, we still need to define better subgroups.

As a result of this study, it was observed that patients with dMMR and TIL-H had a better prognosis. Could this also be used as a predictive factor for determining immunotherapy response? This situation seems to be an area that needs to be studied in terms of both metastatic and adjuvant colon cancer treatments.

\section{CONCLUSION}

Different tumor subtypes could be found with the help of the TIL/MMR-based classification in stage II CC patients with different prognosis. TIL-low/pMMR status is clinically aggressive and TIL-high/dMMR status is a good clinical course phenotype. Therefore, considering that the TIL and MMR evaluated by routine HE staining may be a cost-effective and powerful choice for risk classification in patients with stage II CC. These results support the evaluation and routine use of the combined TIL/MMR status in the treatment plan and follow-up of the patients with stage II CC.

\section{ACKNOWLEDGEMENTS:}

The authors thank Prof. Dr. Kadir Kayatas, and Dr. Mehmet Tepe for their valuable technical assistance and electronic clinical data management.

\section{ETHICAL APPROVAL:}

This study was approved by the Ethical Review Board (ERB) of Haydarpasa Numune Research and Training Hospital, Istanbul, Turkey (Registration No. HNEAH KAEK 2020/KK/15).

\section{PATIENTS' CONSENT:}

Informed consent for this retrospective study was not deemed necessary by the ERB.

\section{CONFLICT OF INTEREST:}

The authors declared no conflict of interest.

\section{AUTHORS' CONTRIBUTION:}

MS: Conception, design, acquistion, analysis, interpretation, data analysis, drafting and revising it critically for important intellectual content and final approval.

EAA, TD: Contributed to design articles and analysing data.

ETS, EC, MC: Contributed to collecting and analysingdata.

MS, EAA, TD, ETS, EC: Contributed to data collection, statistical analysis.

\section{REFERENCES}

1. Sung H, Ferlay J, Siegel RL, Laversanne M, Soerjomataram I,
Jemal A, et al. Global cancer statistics 2020: Globocan estimates of incidence and mortality worldwide for 36 cancers in 185 countries. CA Cancer J Clin 2021; 71(3):209-49. doi: 10.3322/caac.21660.

2. Chen VW, Hsieh MC, Charlton ME, Ruiz BA, Karlitz J, Altekruse SF, et al. Analysis of stage and clinical/prognostic factors for colon and rectal cancer from SEER registries: AJCC and collaborative stage data collection system. Cancer 2014; 120 Suppl 23:3793-806. doi: 10.1002/cncr.29056.

3. Network. NCC. NCCN guidelines: colon cancer, version 2.2020 $2020 \quad$ [Available from: http://www.nccn.org/professionals/physician_gls/pdf/colon.p df.

4. Babcock BD, Aljehani MA, Jabo B, Choi AH, Morgan JW, Selleck MJ, et al. High-risk stage II colon cancer: Not all risks are created equal. Ann Surg Oncol 2018; 25(7):1980-5. doi: 10.1245/s10434-018-6484-8.

5. Huh JW, Lee JH, Kim HR. Prognostic significance of tumorinfiltrating lymphocytes for patients with colorectal cancer. Arch Surg 2012; 147(4):366-72. doi: 10.1001/archsurg.2012.35.

6. Galon J, Costes A, Sanchez-Cabo F, Kirilovsky A, Mlecnik B, Lagorce-Pages $C$, et al. Type, density, and location of immune cells within human colorectal tumors predict clinical outcome. Science 2006; 313(5795):1960-4. doi: 10.1126/science.1129139.

7. Jass JR, Do KA, Simms LA, lino H, Wynter C, Pillay SP, et al. Morphology of sporadic colorectal cancer with DNA replication errors. Gut 1998; 42(5):673-9. doi: 10.1136/gut. 42.5.673.

8. Pages F, Mlecnik B, Marliot F, Bindea G, Ou FS, Bifulco C, et al. International validation of the consensus Immunoscore for the classification of colon cancer: a prognostic and accuracy study. Lancet 2018; 391(10135):2128-39. doi: 10.1016/S0140-6736(18)30789-X.

9. Klintrup K, Makinen JM, Kauppila S, Vare PO, Melkko J, Tuominen $\mathrm{H}$, et al. Inflammation and prognosis in colorectal cancer. Eur J Cancer 2005; 41(17):2645-54.

10. Iseki $Y$, Shibutani M, Maeda K, Nagahara H, Fukuoka T, Matsutani $S$, et al. A new method for evaluating tumor-infiltrating lymphocytes (TILs) in colorectal cancer using hematoxylin and eosin (H-E)-stained tumor sections. PLoS One 2018; 13(4):e0192744. doi: 10.1371/journal.pone.019 2744.

11. Bertagnolli MM, Redston M, Compton CC, Niedzwiecki D, Mayer RJ, Goldberg RM, et al. Microsatellite instability and loss of heterozygosity at chromosomal location 18q: Prospective evaluation of biomarkers for stages II and III colon cancer- a study of CALGB 9581 and 89803. J Clin Oncol 2011; 29(23):3153-62. doi: 10.1200/JCO.2010.33. 0092.

12. Williams DS, Mouradov D, Jorissen RN, Newman MR, Amini E, Nickless DK, et al. Lymphocytic response to tumour and deficient DNA mismatch repair identify subtypes of stage II/III colorectal cancer associated with patient outcomes. Gut 2019; 68(3):465-74. doi: 10.1136/gutjnl-2017-315664.

13. Cha YJ, Park EJ, Baik SH, Lee KY, Kang J. Clinical significance of tumor-infiltrating lymphocytes and neutrophil-to-lymphocyte ratio in patients with stage III colon cancer who under- 
went surgery followed by FOLFOX chemotherapy. Sci Rep 2019; 9(1):11617. doi: 10.1038/s41598-019-48140-1.

14. Lynch HT, Lynch PM, Lanspa SJ, Snyder CL, Lynch JF, Boland CR. Review of the Lynch syndrome: History, molecular genetics, screening, differential diagnosis, and medicolegal ramifications. Clin Genet 2009; 76(1):1-18. doi: 10.1111/j. 1399-0004.2009.01230.x.

15. Rozek LS, Schmit SL, Greenson JK, Tomsho LP, Rennert HS, Rennert G, et al. Tumor-infiltrating lymphocytes, crohn's-like lymphoid reaction, and survival from colorectal cancer. J Natl Cancer Inst 2016; 108(8):djw027. doi: 10.1093/jnci/djw027.

16. Boland $C R$, Goel A. Microsatellite instability in colorectal cancer. Gastroenterol 2010; 138(6):2073-87 e3. doi: 10.1053/j.gastro.2009.12.064.

17. Gkekas I, Novotny J, Fabian P, Nemecek R, Palmqvist R, Strigard $\mathrm{K}$, et al. Deficient mismatch repair as a prognostic marker in stage II colon cancer patients. Eur J Surg Oncol 2019; 45(10):1854-61. doi: 10.1016/j.ejso.2019.05.023.

18. Guastadisegni C, Colafranceschi M, Ottini L, Dogliotti E.
Microsatellite instability as a marker of prognosis and response to therapy: A meta-analysis of colorectal cancer survival data. Eur J Cancer 2010; 46(15):2788-98. doi: 10.1016/j.ejca.2010.05.009.

19. Webber EM, Kauffman TL, O'Connor E, Goddard KA. Systematic review of the predictive effect of MSI status in colorectal cancer patients undergoing 5FU-based chemotherapy. BMC Cancer 2015; 15:156. doi: 10.1186/ s12885-015-1093-4.

20. Gkekas I, Novotny J, Fabian P, Nemecek R, Palmqvist R, Strigard $\mathrm{K}$, et al. Mismatch repair status predicts survival after adjuvant treatment in stage II colon cancer patients. J Surg Oncol 2019. doi: 10.1002/jso.25798.

21. Leijssen LGJ, Dinaux AM, Amri R, Taylor MS, Deshpande V, Bordeianou LG, et al. Impact of intramural and extramural vascular invasion on stage II-III colon cancer outcomes. J Surg Oncol 2019; 119(6):749-57. doi: 10.1002/jso.25367.

22. Andre T, Shiu KK, Kim TW, Jensen BV, Jensen LH, Punt C, et al. Pembrolizumab in microsatellite-instability-high advanced colorectal cancer. N Engl J Med 2020; 383 (23):2207-18. doi: 10.1056/NEJMoa2017699. 